\title{
On Modelling Electricity Spot Prices: A Case Study for the Turkish Market
}

\author{
Ali Ulvi ÖZGÜL ${ }^{1}$ @ , Dündar KÖK²
}

\begin{abstract}
This paper demonstrates the significance of jump threshold in terms of modeling and generating realistic trajectories for an electricity spot price process. Determining the suitable threshold and choosing among distributions proposed in literature are key to the followed moment-matching strategy. This is implemented in a two-factor model framework with the Turkish spot electricity price data. The market studied is a developing one which has taken huge steps in liberalization by learning from more advanced markets, yet with limited research on her spot price dynamics. The selected two-factor model entails downward jumps, which are increasingly getting essential components of the process with the progressing integration of renewable sources. Such components are notably observed since 2015 in the spot market. Moreover, considering structural changes in seasonality both improves the model fit and reveals the same year as the break year for Turkish market. Finally, underlying economic interactions and policy implications for the market are discussed.
\end{abstract}

Keywords: Turkish electricity market; Spot price models; Seasonality modelling; Jump modelling; Seasonality breaks; Jump threshold; Risk management

JEL Classification: C13, C32, C58, G17

\section{INTRODUCTION}

The restructuring of electricity markets which started in the last decade of the preceding century has given rise to increasing interest towards electricity spot price modelling. This is due to not only the idiosyncrasies of the commodity in question and the market but also the proliferation of risk management products pertaining to electricity. Evolving nature of liberal electricity markets in general and the unique features of the spot market in question call for more realistic and convenient models.

Spot electricity markets can be viewed as the result of dual-market model adopted during the restructuring, or liberalization process which affected the electricity markets worldwide. Being a fungible commodity, the same unit of electricity can be substituted with one another provided that time and location of delivery are held constant (Edwards, 2010). This feature has led electricity to follow financialization, which is described by Davis and Kim (2015) as the overwhelming trend characterized by the increasing role of financial markets in our lives as the overwhelming trend characterized by the increasing role of financial markets in our lives. European Energy Exchange AG (EEX), operating various power markets in Europe highlights substantial growth rates in her annual reports. Trading volume of power derivatives reached 3.920 TWh in 2016, 7.3 times the spot market volume. The compounded annual growth rates on derivatives and spot market volumes between 2012 and $2016, \% 43.2$ and $\% 12.1$ respectively (European Energy Exchange AG, 2014, 2017), manifest financialization is already under way and the increasing need for standardized risk management products.

Compared to her more mature counterparts in US and Europe, mainly for which price models are formulated, the Turkish market can be considered as an emerging one having taken substantial steps in restructuring. The market merits such modelling efforts for not only the alleged progress but also the means to identify and address evolving issues. Considering this and the limited research on this market, we aim to find 
the most suitable model for spot prices generated in Turkish electricity market.

The model alternatives are evaluated among those in quantitative finance domain which are driven predominantly by the increasing need for derivatives modelling and pricing. In this context, we restrict modelling efforts to the second group, which uses solely spot prices, in the taxonomy proposed by Aïd (2015). The proposed model and improvements not only constitute the basic step in shifting to other groups of models, potentially offering more realistic portrait by employing forward/futures dynamics, but also helps this emerging market progress by incorporating relevant information to enhance participants' predictions, thereby facilitate efficiency.

Section 2 of this paper gives an outline of the electricity spot markets, specifically the Turkish market, and some leading models from the literature. Section 3 presents the data and time series properties. Seasonal trends and the stochastic component are modelled in Section 4. We propose and implement improvements to the selected two-factor model in Section 5. Finally, Section 6 concludes the paper with discussion of implications.

\section{TURKISH SPOT ELECTRICITY MARKET AND PRICE MODELS}

\subsection{Overview}

Limits and inefficiencies in electricity storage imply uninterrupted balancing requirements at all nodes and times within the electric power system (EPS). System operator, in coordination with the market operator in case the latter is a separate entity as in self-dispatched markets, maintains balanced EPS through spot markets. Spot market, when taken from this point of view, is comprised of three markets with differing delivery horizons and balancing duties: day-ahead, intra-day and balancing (real-time) power markets.

Day-ahead mechanism, as the name implies, serves as the medium where planning for the next day occurs. More than that, like in the Turkish market liberalization process, this mechanism evolves into a market whereby the quantities to be traded and the corresponding prices for the $\mathbf{2 4}$ hours (or 48 half-hours) of the following day are settled. For a specific hour, other two markets meet the balancing requirements which become apparent in time further to finalization of the day-ahead plan.
We will refer to day-ahead market (DAM) as the 'spot market' here following the literature referenced. Although time to delivery is shorter in intra-day market and almost zero in balancing market, DAM is considerably more liquid. This is because of the fine balancing needs in EPS arising from peculiarities of electricity and the increased risks as one approaches the delivery period.

\subsection{Turkish Spot Electricity Market}

The initiation of Turkish spot market can be traced back to day-ahead-planning mechanism which begun in July 2009. Transition to a completely new market, in financial sense, was designed and implemented by having participants adapted to the new system through training programs and experience till DAM became operational on Dec. $1^{\text {st }}, 2011$ (Tokyay \& Ozdemir, 2013). Turkish DAM with around $18 \%$ share in physical electricity markets as of this date has progressed steadily to reach nearly half the volume traded by 2015 as presented in Figure 1 evidencing participants' confidence therein and its significance in the industry.

The market operator, Energy Exchange Istanbul (EXIST; incorporated as Enerji Piyasalari Isletme A.S. (EPIAS) per Turkish Commercial Code) sets the hourly settlement prices by algorithms which aim at maximizing the market surplus (Enerji Piyasalari Isletme A.S., 2016). Hence DAM prices reflecting equilibrium are based on uniform pricing. As opposed to pay-as-bid pricing scheme where market participants are paid according to their accepted bids (Shahidehpour, Yamin, \& Li, 2002), DAM participants in Turkey are paid at/pay the same unit price, i.e., market price which indicates the marginal price of the last unit to be traded.

\subsection{Modelling Framework}

Spot price process $\left(S_{t}\right)$, predominantly in logarithmic form, is assumed to be made up of seasonal and stochastic components,

$$
S_{t}=e^{\Lambda(t)+Z_{t}}
$$

where $\Lambda(t)$ is the deterministic seasonality function and $Z_{t}$ is the stochastic component. Hence any seasonality of stochastic nature passes to the process $Z_{t}$, rendering this virtually impossible to be identified.

The sinusoidal pattern in prices characterized by hills and valleys round the year has almost made sinusoidal functions, i.e., Fourier analysis, the sine qua non of seasonality modelling, though some other methods are also used. Seasonal variations in spot price can be 
filtered by using the function given in Eqn. (2). While the last four terms in this basic form account for yearly and weekly seasonality patterns, the constant and trend terms can be regarded as fixed cost associated with power generation and long-run linear trend in total generation cost (Geman \& Roncoroni, 2006).

$$
\begin{aligned}
& \Lambda(t)=c_{1}+c_{2} t+c_{3} \cos \left(\frac{2 \pi t}{365}\right)+ \\
& c_{4} \sin \left(\frac{2 \pi t}{365}\right)+c_{5} \cos \left(\frac{2 \pi t}{7}\right)+ \\
& c_{6} \sin \left(\frac{2 \pi t}{7}\right)
\end{aligned}
$$

A hybrid approach employing both sinusoidal functions and dummy variable/differencing methods is also adopted to model seasonality in prices.

Jumps and mean reversion are the two dominant characteristics of an electricity spot price process. Jumps, often called 'spikes', by their nature result in separate regime(s). We refer to Harris (2006); Hayfavi \& Talasli (2014); Seifert \& Uhrig-Homburg (2007) for the differentiation between the two terms and various views on the classification of these extreme price changes. Finer classification based on price variations preceding and following a jump leads to more realistic models by addressing class-specific stochastic components, i.e., factors. We prefer to use "jump" to express extreme change in spot prices at a specific time point irrespective of the speed of reversion to normal price level. Once a jump is located, classification can be accomplished jointly by estimating corresponding mean reversion rate and considering extreme moves in its neighbourhood.

Detection of jumps are often carried out by setting a price change threshold which represents a cut-off between normal price variations and extreme ones. Clewlow \& Strickland (2000) used this idea with a recursive filtering method, which has later gained popularity among researchers.

Mean reversion is explained within price equilibrium setting in which relatively high prices induce higher-cost suppliers to enter the market, subsequently leading to downward pressure on prices due to excess supply. The same reasoning holds for prices which are lower than the equilibrium price, hence tendency towards mean price level is expected to be common in commodity prices (Schwartz, 1997). Although there are opposing views and evidence as to mean reversion in other commodities, it is a dominant feature of electricity price process, particularly due to much greater reversion force in action.

Ornstein-Uhlenbeck (OU) process, which is widely used to model mean reversion, is defined to be the
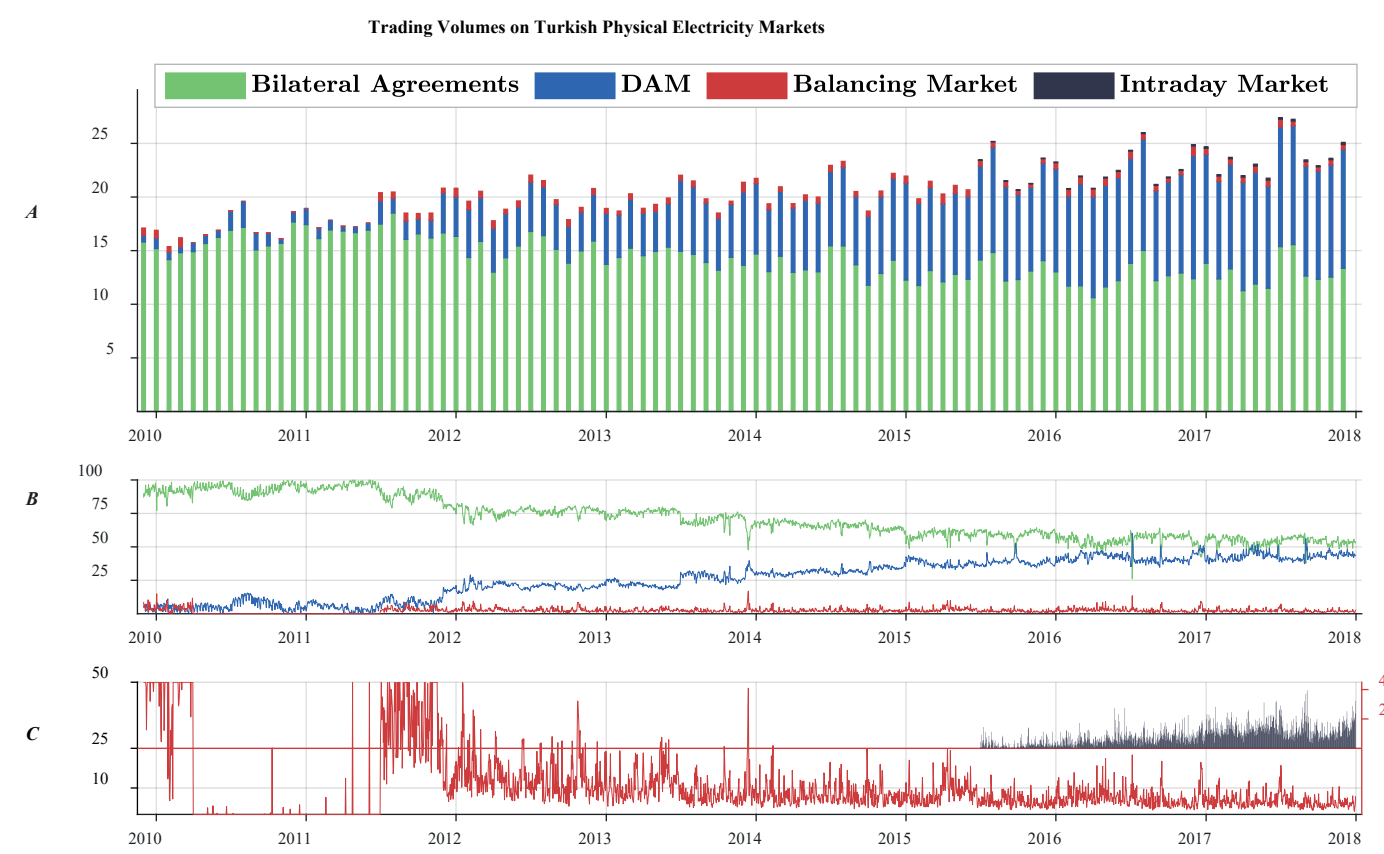

A. Monthly figures in TWh, B. \% in total (daily), C. Normalized by DAM figures (\%, daily).

Figure 1: The evolution of Turkish physical electricity markets (12/2009-12/2018). 
process $X_{t}$ which solves the stochastic differential equation:

$$
d X_{t}=-\lambda X_{t} d t+d z_{t}
$$

This definition is a general one since $z_{t}$ is a Lévy process starting at 0 . Brownian motion is the sole member that has no jump within the family of such processes. The processes defined through Eqn. (3) having a stochastic component $\left(X_{t}\right)$ other than Brownian motion, which are known as Non-Gaussian OU processes, provide flexibility in modelling dependencies and capture divergences from Gaussianity (Barndorff-Nielsen \& Shephard, 2001). That is why they have notable contributions in developing multi-factor models in electricity price modelling.

\subsection{Electricity Spot Price Models}

Lucia \& Schwartz (2002) have conducted the first comprehensive research on both electricity spot and forward/futures price dynamics. Along with single factor model, the authors also suggest a two-factor model. Demonstrated superiority of sinusoidal function in capturing yearly seasonality over monthly dummies, i.e., step function approach, is an outstanding contribution of this paper.

The authors acknowledge that additional factor for jumps can be incorporated into the model. This idea has led to the popular mean-reverting jump diffusion (MRJD) models. Some sophisticated versions of MRJD models are implemented by Cartea \& Figueroa (2005) and Seifert \& Uhrig-Homburg (2007) .

Among the more advanced models, those developed by Geman \& Roncoroni (2006) and Benth, Kallsen, \& Meyer-Brandis (2007) are worth considering. Besides portraying the spot prices realistically, the difference equation in Geman and Roncoroni (GR) Model proves to be useful in derivatives pricing since it is based on real probability measure yet succeeds to preserve the Markov property in its proposed solution. What makes GR Model so special is its modelling approach towards matching the moments of the empirical distribution as originated in the market. By choosing the most suitable values for price change and regime switching thresholds, the authors achieved the optimum values for parameters and obtained realistic trajectories for the markets with varying price behaviours, i.e., level of "pressure". The model also considers probable periodicity in jump behaviour through the suggested intensity process.
Benth, Kallsen \& Meyer-Brandis (BKM) Model postulates that price process can be modelled as a weighted sum of several factors, which move by jumps with their own mean reversion rates. We refer to original article for technical details and the derived derivatives pricing equations (Benth et al., 2007). Since the authors have not calibrated the model with real market data, we direct readers to Meyer-Brandis \& Tankov (2008), Klüppelberg, Meyer-Brandis, \& Schmidt (2010) and Gonzalez, Moriarty, \& Palczewski (2017) for practical applications.

\section{DATA \& PRELIMINARY ANALYSIS}

We have retrieved hourly DAM prices settled and announced by the market operator (EXIST) for the period 01/01/2012-31/12/2017. Taking daily averages, we obtain spot price series. Data frequency and the market chosen to represent spot market are in line with the models reviewed above.

Spot prices are denominated in Turkish Lira (TL) per megawatt hour ( $M W h$ ) as can be seen from Figure 2 and this corresponds to the price for 10 lots. The starting point for time series coincides with the commencement of Turkish DAM.

\subsection{Statistical Features}

Like other financial time series, both spot price series and its log-transformed variant are far from normality. Transformation somewhat compresses data. This can be deduced from Table 1 by comparing normalized ranges for two series, which are 20.15 and 15.54 for Spot and logSpot, respectively. While compression brings series closer to normality, it distorts the series by shifting the empirical distribution to the right.

Cited distortion in log-transformation has a more important implication for jump modelling. Downward extreme price moves from the base regime have emerged as a market fact due to increasing integration of renewable generation into market mechanism. Feed-in tariffs and similar subsidies resulting from economic and environmental concerns have resulted in more investments, in turn increasing share of renewables in total generation. This trend has started to create downward pressure in prices due to merit-order effect as argued by Grossi, Heim, Hüschelrath, \& Waterson (2018). We also note that magnitudes of these downward jumps are artificially escalated by the log-transformation, which also explains the shift in distribution. This effect can be visualized through Figure 2 by comparing Spot and logSpot series. 


\subsection{Stationarity of Price Series}

Due to strong mean-reversion of electricity spot prices, researchers have focused on more complicated facets of modelling. While Meyer-Brandis \& Tankov (2008) has shown evidence against unit root on the raw and deseasonalized spot price series of the six major electricity markets, they describe jumps as the non-stationary component of electricity price process has shown evidence against unit root on the raw and deseasonalized spot price series of the six major electricity markets, they describe jumps as the non-stationary component of electricity price process. This identification clearly rests upon the deteriorating impact of jumps on distributional properties.

We report Augmented Dickey-Fuller (ADF), Phillips-Perron (PP) unit root and Kwiatkowski-Phillips-Schmidt-Shin (KPSS) stationarity test results on Table 1 for a comprehensive treatment of Turkish spot price series. Unit root hypotheses are clearly rejected by ADF tests for Spot and logSpot. KPSS tests partially confirm this finding.

Stationarity can be rejected with the version accompanying both constant and trend terms. Having obtained contradicting results for hourly price series, Popova (2008) rates KPSS as a useful tool, yet this functionality is meaningful when its results are evaluated with those of its alternatives. To this end, Den Haan and Levin note the sensitivity of inferences through KPSS in terms of variance estimators employed (Popova, 2008). PP tests, in this account verify the ADF results, hence we conclude our series are stationary owing to significant mean reversion.

\section{EMPIRICAL ANALYSIS}

The benchmark models reviewed try to describe evolution of price series with its own dynamics, within time series analysis framework. Sticking with this approach we model season effects and stochastic component in the sequel.

\subsection{Analysis of Seasonal Trend}

We follow Eqn. (1); therefore, deterministic seasonality function will be fitted to logSpot series. This is equivalent to adopting arithmetic seasonality as opposed to multiplicative one, advocated by Meyer-Brandis \& Tankov (2008) This is equivalent to adopting arithmetic seasonality as opposed to multiplicative one, advoca-

Table 1: Descriptive and unit root/stationarity test statistics for Turkish spot (Spot) and logarithmic spot (logSpot) electricity price series.

\begin{tabular}{|c|c|c|c|}
\hline & & Spot & logSpot \\
\hline \multicolumn{4}{|l|}{ Panel A: Descriptive statistics } \\
\hline Mean & & 151.02 & 4.99 \\
\hline Median & & 151.20 & 5.02 \\
\hline Minimum & & 22.93 & 3.13 \\
\hline Maximum & & 687.00 & 6.53 \\
\hline Std. deviation & & 32.96 & 0.22 \\
\hline Skewness & & 3.16 & -1.13 \\
\hline Kurtosis & & 50.09 & 11.50 \\
\hline Jarque-Bera (JB) statistics & & 206.20 & 7.06 \\
\hline \multicolumn{4}{|c|}{$\begin{array}{l}\text { Panel B: Augmented Dickey-Fuller (ADF), Kwiatkowski-Phillips-Schmidt-Shin (KPSS) and Phillips- } \\
\text { Perron (PP) test results. }\end{array}$} \\
\hline ADF with constant term & $(21,-3.4369,-2.5683)$ & -5.0881 & -4.9367 \\
\hline ADF with constant and trend terms & $(21,-3.9679,-3.1283)$ & -5.1159 & -4.9578 \\
\hline KPSS with constant term & $(32,0.7390,0.3470)$ & 0.2438 & 0.2618 \\
\hline KPSS with constant and trend terms & $(33,0.2160,0.1190)$ & 0.2299 & 0.2654 \\
\hline PP with constant term & $(30,-3.4331,-2.5674)$ & -28.0893 & -23.5588 \\
\hline PP with constant and trend terms & $(30,-3.9622,-3.1278)$ & -28.1282 & -23.5852 \\
\hline
\end{tabular}

Numbers in parentheses indicate optimum lag lengths, critical values at $1 \%$ and $5 \%$ significance levels respectively for the tests in corresponding rows. Optimum lag length is determined by Schwarz-Bayesian information criterion for ADF tests. Bartlett kernel and Newey-West automatic selection method is used for KPSS and PP tests. 
ted by Meyer-Brandis \& Tankov (2008) to circumvent the side effects of the log-transformation to circumvent the side effects of the log-transformation. The overall, annual, and weekly patterns in our series can be modelled through Eqn. (4); the last two terms being reserved for day effects, i.e., weekly pattern. Likewise, the model can be extended with the terms for semi-annual and quarterly trends [Eqn. (5) \& Eqn. (6)]. We represent three models in vector form below $\left(\Lambda^{i}, i=1,2,3\right)$ :

$$
\begin{aligned}
\Lambda^{1}(\mathrm{t}) & =\left[\begin{array}{llll}
\mathbb{1} & \mathrm{t} & \mathrm{Y} & \mathrm{W}
\end{array}\right] \beta^{1^{\prime}} \\
\Lambda^{2}(t) & =\left[\begin{array}{lllll}
\mathbb{1} & t & Y & S & W
\end{array}\right] \beta^{2^{\prime}} \\
\Lambda^{3}(t) & =\left[\begin{array}{llllll}
\mathbb{1} & t & Y & S & Q & W
\end{array}\right] \beta^{3^{\prime}}
\end{aligned}
$$

where $\forall t=1, \cdots, N$; yearly $(Y)$, semiannual $(S)$, quarterly $(Q)$ and weekly $(W)$ trends are defined as:

$$
\begin{aligned}
& \mathrm{Y}(t)=\left[\begin{array}{lll}
\cos \left(\frac{2 \pi \mathrm{t}}{365}\right) & \sin \left(\frac{2 \pi \mathrm{t}}{365}\right)
\end{array}\right], \quad \mathrm{S}(t)=\left[\begin{array}{ll}
\cos \left(\frac{4 \pi \mathrm{t}}{365}\right) & \sin \left(\frac{4 \pi \mathrm{t}}{365}\right)
\end{array}\right] \\
& \mathrm{Q}(t)=\left[\begin{array}{ll}
\cos \left(\frac{8 \pi \mathrm{t}}{365}\right) & \sin \left(\frac{8 \pi \mathrm{t}}{365}\right)
\end{array}\right], \quad \mathrm{W}(t)=\left[\begin{array}{ll}
\cos \left(\frac{2 \pi t}{7}\right) & \sin \left(\frac{2 \pi t}{7}\right)
\end{array}\right]
\end{aligned}
$$

and $\mathbb{1}, t$ denote corresponding row elements of vector of ones and trend vector, respectively. Since other methods for daily effects are covered next, we exclude the final component $(W)$ and denote the resulting models by $S^{1}, S^{2}$ and $S^{3}$ which remain intact through analysis.

For a possible better fit, we implement differencing and dummy variable approaches. While all day types, i.e., weekdays and holidays, are incorporated in differencing method, similar days in terms of price behaviour can be accounted for as a group in the latter one.

Following De Jong (2006), we label our data in terms of weekdays $(1, \cdots, 7)$ to assess price differentiation due to day effects. Labels for the days which correspond to official holidays in Turkey throughout the period are changed to " 8 ". Deviations from overall average price $\left(\mu_{S}\right)$ are calculated for each day type and subsequently normalized to keep series in place through:

$$
\begin{array}{rlrl}
d_{k} & =\mu_{k}-\mu_{S} & k & =1, \ldots, 8 \\
w_{k} & =d_{k}-\frac{\sum_{i=1}^{k} d_{i}}{k} & k & =1, \ldots, 8
\end{array}
$$
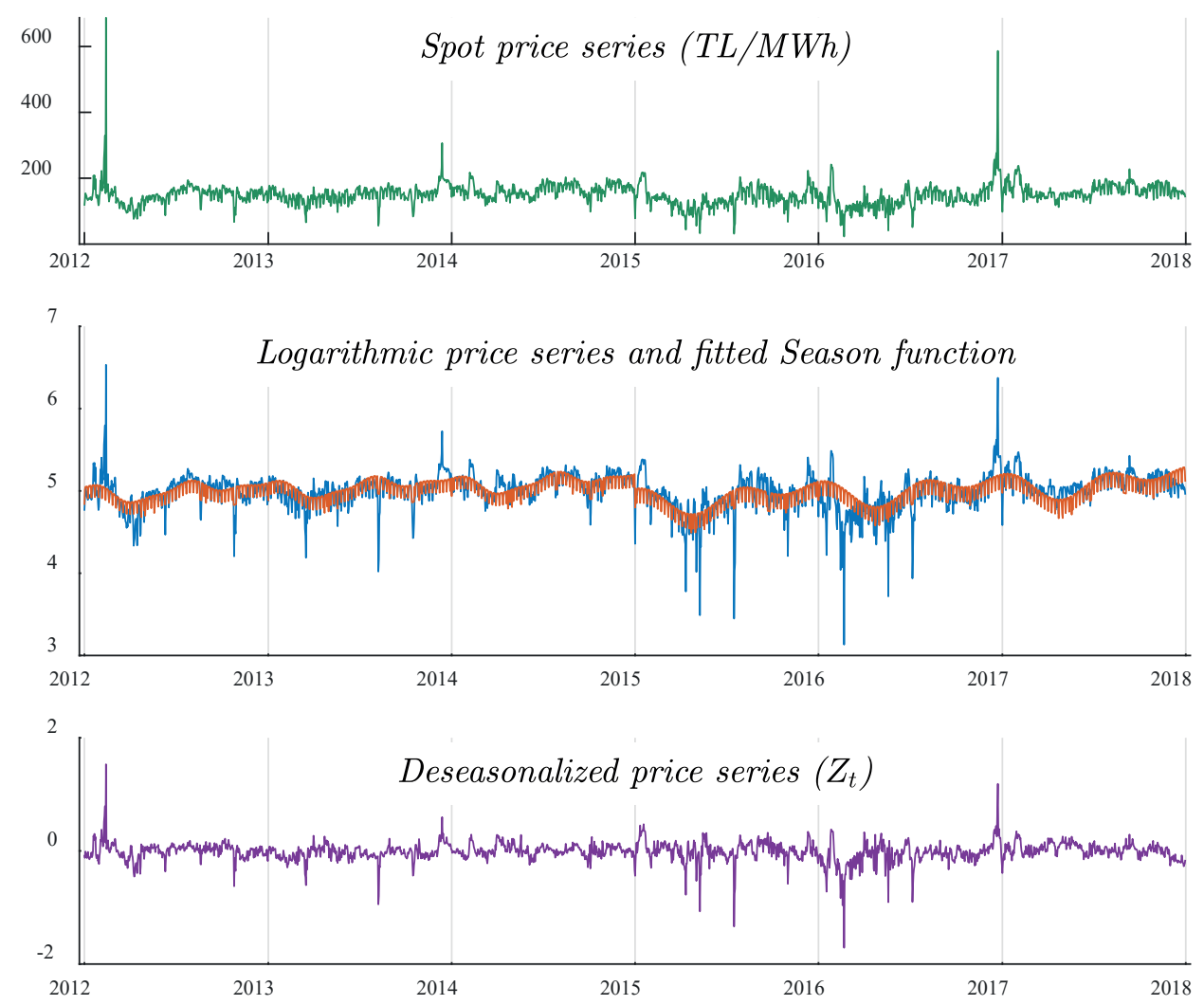

Figure 2: Spot electricity price series, logarithmic price series (logSpot) with the fitted season function and the deseasonalized (stochastic) series, $\left(Z_{t}\right)$. 
Similar calculation can be made separately for each year rather than taking the series in whole. Results of such analysis are presented visually in Figure 3 for Spot series. Since figures are denominated in the effective currency, i.e., TL/MWh, \pm deviations per weekdays and holidays have straightforward practical implication for trading desks. As far as deterministic function is concerned, corresponding deviation for a day type $\left(w_{k}\right)$ must be subtracted to offset day type effect. Equivalently,

$$
\Lambda^{* 1}(\mathrm{t})=w_{t}+\left[\begin{array}{lll}
\mathbb{1} & \mathrm{t} & \mathrm{Y}
\end{array}\right] \beta^{* 1^{\prime}}
$$

where $w_{t}=w_{k} \Leftrightarrow \operatorname{day}(t)=k, \quad k=1, \cdots, 8$ and day $(t)$ corresponds to label function we use for day types. The same modification also applies to $\Lambda^{* 2}$ and $\Lambda^{* 3}$.

A remarkable finding through this method is the nature of Saturday series in explaining the overall average price. The same representation somewhat applies to Monday series. Likewise, certain day types can be treated in clusters, e.g., Tuesdays with Wednesdays and/or Fridays. Such clustering makes dummy variable approach more convenient; in anticipation we also consider three alternative dummy models:

$$
\begin{aligned}
D M^{1} & =\left[\begin{array}{llll}
D_{\text {Sun }} & D_{\text {Tue }, \text { Wed,Thu,Fri }} & D_{\text {Hol }}
\end{array}\right], \\
D M^{2} & =\left[\begin{array}{llll}
D_{\text {Sun }} & D_{\text {Tue }, \text { Wed,Fri }} & D_{\text {Thu }} & D_{\text {Hol }}
\end{array}\right], \\
D M^{3} & =\left[\begin{array}{lllll}
D_{\text {Sun }} & D_{\text {Tue }, \text { Fri }} & D_{\text {Wed }} & D_{\text {Thu }} & D_{\text {Hol }}
\end{array}\right],
\end{aligned}
$$

The deterministic function with the first dummy model alternative when combined with Model 1 for macro seasonality looks like:

$$
\Lambda^{* * 1}(\mathrm{t})=\left[\begin{array}{llll}
\mathbb{1} & D M^{1} & \mathrm{t} & \mathrm{Y}
\end{array}\right] \beta^{* * 1^{\prime}}
$$

We follow the iterative procedure of Klüppelberg et al. (2010) to mitigate the weakening impact of outliers on estimators. In this respect, Robust Least Squares (RLS) estimation is conducted for each variant of season model specifications conceived. Denoting the standard deviation of residuals by $s$, the observations in the interval $[-1.5 s, 1.5 s]$ are left unchanged, while those outside this region are set equal to the nearest end point at each iteration. The recursive procedure ends when the season functions in successive iterations sufficiently converge to each other. Convergence is checked by summing squared differences between the values mapped by two season functions. We choose this sum to be less than 0.001 at the earliest as a stopping criterion and note that this choice does not cause any marginal delay in convergence, and brings
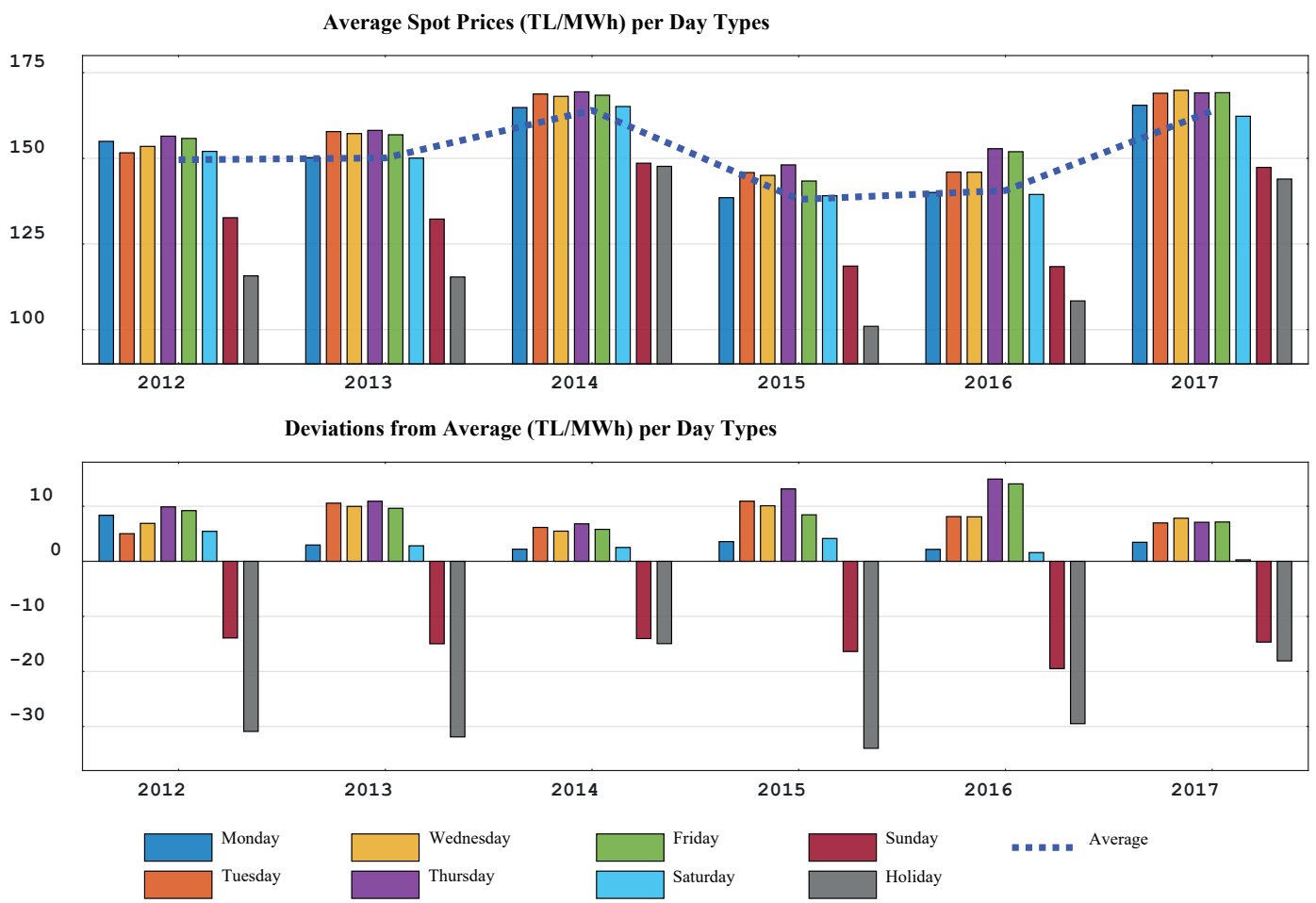

Figure 3: Weekday effects on Turkish spot electricity prices, nominal in TL/MWh. 
quality of fit and the fewer number of parameters required. Finally, models which include component for quarterly seasonality yield better results for Turkish market if they are implemented within structural break context.

The parameters of the selected model, which are statistically significant at $1 \%$ significance level, are reported in Table 3. We favour models with a single break since results indicate the final year in our series as the second break year and improvement is marginal. Fitted function and residual series of the model can be visualized through Figure 2.

\subsection{Modelling Stochastic Component}

Taking downward jumps which are increasingly observed as a market fact, more recent studies (see Gonzalez et al., 2017; Mayer, Schmid, \& Weber, 2015) Taking downward jumps which are increasingly observed as a market fact, more recent studies (see Gonzalez et al., 2017; Mayer, Schmid, \& Weber, 2015) accommodate these in their modelling frameworks, and they purposely cover major European electricity markets, which are greatly experiencing the transition due to integration of renewables accommodate these in their modelling frameworks, and they purposely cover major European electricity markets, which are greatly experiencing the transition due to integration of renewables. While the latter is an implementation of BKM Model in two and alternatively three factors settings (2OU and 3OU) through Bayesian calibration, the other proposes a two-factor model, which will be referred to as Mayer-Schmid-Weber (MSW) Model. The model is based on some theoretical results and insights of the advanced models reviewed earlier.

On the other hand, downward jumps also impose limitations on implementing GR Model. Three possible

Table 3: Summary for the final seasonality model (logSpot). All estimations are based on Robust Least Squares.

\begin{tabular}{|c|c|c|c|c|c|c|c|c|c|c|c|}
\hline \multicolumn{12}{|c|}{ Model for the First Period $\left(2012-2014, \widehat{\Lambda}_{1}\right)$} \\
\hline & $\widehat{\boldsymbol{\beta}}_{1}$ & $\widehat{\boldsymbol{\beta}}_{2}$ & $\widehat{\boldsymbol{\beta}}_{3}$ & $\widehat{\boldsymbol{\beta}}_{4}$ & $\widehat{\boldsymbol{\beta}}_{5}$ & $\widehat{\boldsymbol{\beta}}_{6}$ & $\widehat{\boldsymbol{\beta}}_{7}$ & $\widehat{\boldsymbol{\beta}}_{8}$ & $\widehat{\boldsymbol{\beta}}_{9}$ & $\widehat{\boldsymbol{\beta}}_{10}$ & $\widehat{\boldsymbol{\beta}}_{11}$ \\
\hline Coefficients & 4.9585 & -0.1157 & 0.0330 & - & 0.0001 & 0.0142 & - & 0.0601 & 0.0388 & - & 0.0175 \\
\hline \multirow[t]{3}{*}{ t-Stats } & 524.83 & -9.79 & 3.93 & -9.61 & 12.19 & 2.80 & -10.96 & 11.83 & 7.61 & -4.21 & 3.41 \\
\hline & $\mathbf{R}^{2}$ & Adj. $R^{2}$ & MSE & RMSE & LL & AIC & SC & HQ & & & \\
\hline & 0.4093 & 0.4039 & 0.0185 & 0.1361 & 635.98 & -1.1405 & -1.0903 & -1.1215 & & & \\
\hline \multicolumn{12}{|c|}{ Model for the Second Period $\left(2015-2017, \widehat{\Lambda}_{2}\right)$} \\
\hline & $\widehat{\beta}_{1}$ & $\widehat{\boldsymbol{\beta}}_{2}$ & $\widehat{\boldsymbol{\beta}}_{3}$ & $\widehat{\boldsymbol{\beta}}_{4}$ & $\widehat{\boldsymbol{\beta}}_{5}$ & $\widehat{\boldsymbol{\beta}}_{6}$ & $\widehat{\boldsymbol{\beta}}_{7}$ & $\widehat{\boldsymbol{\beta}}_{8}$ & $\widehat{\boldsymbol{\beta}}_{9}$ & & \\
\hline Coefficients & 4.8499 & -0.1334 & 0.0447 & -0.1834 & 0.0002 & 0.0554 & -0.0842 & 0.0745 & 0.0741 & & \\
\hline \multirow[t]{3}{*}{ t-Stats } & 426.49 & -9.25 & 4.41 & -8.13 & 16.93 & 8.98 & -13.18 & 12.13 & 11.96 & & \\
\hline & $\mathbf{R}^{2}$ & Adj. $R^{2}$ & MSE & RMSE & LL & AIC & SC & HQ & & & \\
\hline & 0.4264 & 0.4221 & 0.0367 & 0.1915 & 260.73 & -0.4594 & -0.4183 & -0.4438 & & & \\
\hline \multicolumn{12}{|c|}{ Statistics for the Seasonality Model in Aggregate } \\
\hline & $\mathbf{R}^{2}$ & Adj. $R^{2}$ & MSE & RMSE & LL & AIC & SC & HQ & & & \\
\hline & 0.4313 & 0.4263 & 0.0276 & 0.1662 & 833.69 & -0.7424 & -0.6905 & -0.7234 & & & \\
\hline \multicolumn{12}{|c|}{$\begin{array}{l}\widehat{\Lambda_{1}} \\
=\widehat{\beta_{1}}+\widehat{\beta_{2}} D_{S}+\widehat{\beta_{3}} D_{T u, W, T h, F}+\widehat{\beta_{4}} D_{H}+\widehat{\beta_{5}} t+\widehat{\beta_{6}} \cos \left(\frac{2 \pi t}{365}\right)+\widehat{\beta_{7}} \sin \left(\frac{2 \pi t}{365}\right)+\widehat{\beta_{8}} \cos \left(\frac{4 \pi t}{365}\right) \\
+\widehat{\beta_{9}} \sin \left(\frac{4 \pi t}{365}\right)+\widehat{\beta_{10}} \cos \left(\frac{8 \pi t}{365}\right)+\widehat{\beta_{11}} \sin \left(\frac{8 \pi t}{365}\right) \\
\widehat{\Lambda_{2}} \\
=\widehat{\beta_{1}}+\widehat{\beta_{2}} D_{S}+\widehat{\beta_{3}} D_{T u, W, T h, F}+\widehat{\beta_{4}} D_{H}+\widehat{\beta_{5}} t+\widehat{\beta_{6}} \cos \left(\frac{2 \pi t}{365}\right)+\widehat{\beta_{7}} \sin \left(\frac{2 \pi t}{365}\right)+\widehat{\beta_{8}} \cos \left(\frac{4 \pi t}{365}\right) \\
+\widehat{\beta_{9}} \sin \left(\frac{4 \pi t}{365}\right)\end{array}$} \\
\hline
\end{tabular}


remedies deserve attention to overcome the restriction. Switching to multiplicative seasonality helps eliminate artificially inflated portion of jumps, yet does not rule out those inherent in data generating mechanism. However, the model is based on arithmetic seasonality. Secondly, following the literature (e.g., Geman \& Roncoroni, 2006; Hayfavi \& Talasli, 2014; Klüppelberg et al., 2010; Pirino \& Renò, 2010) weekends can be removed from the series. Such exclusion has been justified by Klüppelberg et al. (2010) through weekends' in capacity to invoke any appealing statistical features. Jumps are the most remarkable among such features and the ones we detect seem to occur more often on Saturdays than weekdays. The order statistics we present in Table 4 also justify this end. Moreover, downward price jumps are predominantly observed on holidays and weekends. We accordingly retain the whole series, which is also consistent with pricing some derivatives which do not make any distinction between day types, i.e., weekly, monthly futures contracts. The ultimate alternative would be augmenting the model to account for downward jumps. Yet, this requires derivation of a new likelihood function, asking for considerable theoretical work.

Although not explicit parameters of the likelihood function in GR Model, thresholds determine the boundaries between continuous part of the process and jumps. Bayesian methods, and Markov regime-switching models in this vein, adopt similar approach and allow relevant parameters to change for the best fit. Further to calibrating MSW Model with its original threshold, we proceed with improvements through proposed simulation methodology.

\subsection{MSW Model Implementation}

Mayer et al. (2015) associate limited option trading on electricity with lack of sufficient information on spot price process associate limited option trading on electricity with lack of sufficient information on spot price process. They, in attempt to fill this gap, propose an underlying spot price model which combines mean reversion, jumps, stochastic volatility and negative prices. Assuming $\left(\Omega, P, \mathcal{F},\left\{\mathcal{F}_{t}\right\}_{t \in[0, T}\right]$ is a complete filtered probability space, where $T<\infty$ is a fixed time horizon, the spot price process $S_{t}$ is governed by:

$$
S_{t}=e^{\Lambda(t)+X_{t}+Y_{t}}
$$

The stochastic Lévy processes, $X_{t}$ and $Y_{t}$ which are independent of each other, describe normal price behaviour and jumps respectively, and follow the OU processes:

$$
\begin{aligned}
& d X_{t}=-\alpha_{X} X_{t} d t+\sigma(t) d B_{t} \\
& d Y_{t}=-\alpha_{Y} Y_{t} d t+d I_{t}
\end{aligned}
$$

with corresponding reversion rates $\alpha_{X}$ and $\alpha_{Y}$, where $d B_{t}$ denotes increments of the Wiener process and $d I_{t}$ stands for compound Poisson process. The model also allows for time-varying volatility in the diffusion process.

Since DAM price for any hour is not allowed to be negative in Turkish market, we ignore the additional

\begin{tabular}{|c|c|c|c|c|}
\hline \multirow{2}{*}{ Day Type } & \multicolumn{2}{|c|}{ Lowest Observations } & \multicolumn{2}{|c|}{ Highest Observations } \\
\hline & logSpot & $Z_{t}$ & $\operatorname{logSpot}$ & $Z_{t}$ \\
\hline Monday & 10 & 6 & 7 & 4 \\
\hline Tuesday & 2 & 5 & 5 & 5 \\
\hline Wednesday & 1 & 3 & 7 & 4 \\
\hline Thursday & 2 & 1 & 9 & 8 \\
\hline Friday & 3 & 6 & 10 & 8 \\
\hline Saturday & 5 & 6 & 9 & 10 \\
\hline Sunday & 15 & 10 & 3 & 8 \\
\hline Holiday & 12 & 13 & $\mathbf{0}$ & 3 \\
\hline
\end{tabular}

Table 4: Number of highest and lowest 50 observations each by day type for logSpot and the stochastic (deseasonalized) series $\left(\mathrm{Z}_{\mathrm{t}}\right)$. 
term of the original model for negative prices. The jump process $I_{t}$ has two components, one for positive and the other for negative jumps:

$$
I_{t}=I_{t}^{+}-I_{t}^{-}
$$

Both jump components are specified by Eqn. (13), with counting processes $N^{ \pm}$and jump intensities $\lambda^{ \pm}$. Jump sizes $J_{i}^{ \pm}$are lognormally distributed with parameters $\mu^{ \pm}$and $\sigma^{ \pm}$.

$$
\begin{aligned}
& I^{ \pm}=\sum_{i=1}^{N_{t}^{ \pm}} J_{i}^{ \pm} \quad \text { s.t. } \quad N^{ \pm}(0)=0 \\
& \mathbb{E}\left(N^{ \pm}\right)=\operatorname{Var}\left(N^{ \pm}\right)=\lambda^{ \pm} \times t \\
& \ln \left(J_{i}^{ \pm}\right) \sim \mathcal{N}\left(\mu^{ \pm}, \sigma^{ \pm}\right)
\end{aligned}
$$

The crucial idea for calibration rests on offsetting mean reversion effect(s) in the first place during the decomposition of the stochastic components off the whole process. This conception resembles the methodology suggested by Geman \& Roncoroni (2006) in estimating Brownian volatility for normal regime. Similarly, the idea is augmented by Benth, Šaltyte-Benth, \& Koekebakker (2008) in a one factor (1OU) model context. Mayer et al. (2015) extend the latter approach in their two-reversion model further.

Model estimation is initiated with an improved version of jump filtering proposed by Clewlow \& Strickland (2000). Rather than return series itself, the residuals obtained by regressing this $\left(d Z_{t}\right)$ on lagged deseasonalized series $\left(Z_{t-1}\right)$ serve as input for the subsequent iterative filtering procedure. Estimated coefficient from the linear regression is the reversion parameter for the total process. More complicated recursive procedure comprising the second step of calibration starts with this initial parameter $\hat{\alpha}_{Z_{0}}\left(\hat{\alpha}_{X_{0}}=\hat{\alpha}_{Y_{0}}=\hat{\alpha}_{Z_{0}}\right.$ initially) and the jump vector $\left(\hat{\xi}_{0}\right)$. Each iteration is activated by identifying the preliminary assignments for the stochastic factors, $\sigma d B_{p}$ and $d I_{p}$. Price changes at each time step are totally attributed to either $\sigma d B_{p}$, which is the case in normal regime, or to $d I_{p}$.

The iterative procedure of the second step starts with forming/updating $\sigma d B, d I, X$ and $Y$ series. Update equations for $\sigma d B$ and $d I$ correct $\sigma d B_{p}$ and $d I_{p}$ for corresponding mean reversion effects to prevent shifts in $Z_{t}$. Equations for $X$ and $Y$ are discretized versions of those in Eqn. (11). Jump vector is then updated taking the new residual series, i.e., $\sigma d B+d I$ and the same threshold. This is different than the earlier iterative filtering since it is performed with a single pass where residuals falling outside the region specified by upper and lower bands at plus/minus threshold times the standard deviation of $\sigma d B$ are detected as jump points. A specification from GARCH family here enables moving limits and stochastic volatility for the diffusion. Reversion rates for estimated $X$ and $Y$ processes are updated next by similar regressions of the first step. The outputs of the iteration, i.e., updated jump vector and estimated reversion rates of the subprocesses are transmitted to the next one.

The original algorithm prescribes convergence in reversion rates as the stopping rule. We consider the change in both $\alpha_{X}$ and $\alpha_{Y}$ at each iteration, take the maximum of these in absolute terms and stop when this value first falls below 0.0002 . Results of calibration are presented in Table 5. We note that the moments of the diffusion process are in line with the normal distribution and JB test confirms this end.

\section{MODEL IMPROVEMENTS}

\subsection{Outline for Model Improvements}

The choice of threshold, i.e., 2.57, applicable in both steps of calibration reflects the assumption that normal price variations, which are governed by Gaussian distribution, make up $99 \%$ of observations at the initial step. Threshold value varies across studies; 2.3 for Turkish market in Talasli (2012), 3 in Clewlow \& Strickland (2000) and Pirino \& Renò (2010). The authors of the latter study also assert that their choice has become customary in this line of research.

Threshold choice has a direct effect on the jump intensity. Stepping threshold values starting from the original up to 5 yields exponentially decaying number of jumps in our experiments. The upper limit pertains to the non-parametric jump detection test suggested for high-frequency time series by Lee \& Mykland (2008). This test proposes a threshold value of 5.13 for our series. However, the data is of daily frequency and higher values substantially reduce jump intensity. On the other hand, intensities estimated by Mayer et al. (2015) are relatively higher in comparison to those found out by empirical counterparts of reference models for the same and comparable markets.

For a better evaluation of the effect threshold has on distributional parameters, i.e., primarily kurtosis, then variance in our case, we simulate 5000 series each with selected thresholds. Additionally, having reviewed various distributions for jump sizes, Exponential (Exp) and Weibull (Weib) distributions are also entertained. Summarizing our simulation experiment, Figure 4 
helps to visualize the effect of threshold and jump size distribution on kurtosis. The kurtosis axis is truncated at 30 since moving to the left on the distribution axis with mid-to-higher thresholds results in considerably larger values than the benchmark. On the other hand, distribution pairs on the right-hand side fail to succeed in approaching this target even at high thresholds, therefore they will be withdrawn in subsequent analysis. We note that the original pair adopted in MSW Model, i.e., Lognormal $(\log N)$ for both types of jumps, belongs to this set.

\subsection{Simulation Methodology}

We augment the MC simulation algorithm of Geman \& Roncoroni (2006) to encompass MSW Model's specifications in discrete time and complement by a probabilistic approach for determining upcoming jump direction. Instead of assigning equal probability to jump occurrence, time-dependent jump intensity functions are fitted for the two subperiods identified in seasonality analysis. Function values at jump points are collectively set as maximum as possible to assign higher probability to distinguished jump events. The acceptance-rejection method customized for such intensity functions is implemented to generate jump times compatible with the implied periodicity (see Geman and Roncoroni, 2006).

Reproducing subprocesses is quite straightforward by using the discrete analogue of update equations. However, determining the direction of jumps at simulated times is a more elaborate task. Sign change threshold happens to be cumbersome here, since a non-reverting jump, i.e., that originating from normal price regime, is likely to be oriented upwards as well as downwards. For simulation purposes, we assume jump direction probability depends on the level of deseasonalized price and the jump sign of the previous observation. Accordingly, a probability matrix conditional on the previous observation through 40 price bins and jump signs $(-1,0,1)$ is instrumented. We find out that fine-tuning the matrix by adding more slices beyond 35-40 has marginal effect on the result. For the sake of consistency, break year has been considered for possible changes in probabilities.

\subsection{Improved Model for the Turkish Market}

A careful examination of Figure 4 (a) suggests narrowing threshold range and eliminating some jump distribution pairs. Accordingly, we set range for threshold as [2.57,4], discard some pairs and add Generalized Pareto (GPar) to the distribution set. By evaluating performance of newly simulated series in matching the fourth and second moments of empirical distribution through graphical analysis, we have identified that four pairs (GPar-Exp, LogN-Exp, Weib-Exp and Exp-LogN) are worth further consideration with suitable thresholds, centred around 2.75, 2.75, 2.78 and 3.89. The latter pair is included to assess impact of higher thresholds on calibration.

Reproducing 10000 series each for these pairs with thresholds in the vicinity of these centres reveals that MSW Model specified by Lognormal and Exponential

Table 5: Summaries of original and improved versions of Mayer-Schmid-Weber (MSW) Model for the Turkish spot electricity market.

Panel A: MSW model output with the original threshold, 2.57. Jump size distributions are lognormal for both positive and negative jumps.

\begin{tabular}{lrrrrrr}
\hline \multirow{2}{*}{ Number of Jumps } & Total & $(+)$ & $(-)$ & Reversion Rates & $\hat{\alpha}_{X}$ & $\hat{\alpha}_{Y}$ \\
& 204 & 91 & 113 & $\hat{\mu}^{-}$ & $\hat{\sigma}^{-}$ & $\hat{\lambda}^{-}$ \\
\hline \multirow{2}{*}{ Jump Distribution Parameters } & $\hat{\mu}^{+}$ & $\hat{\sigma}^{+}$ & $\hat{\lambda}^{+}$ & -1886 & 0.4304 \\
& -1.5002 & 0.3630 & 0.0415 & -1.3570 & 0.4659 & 0.0516 \\
\hline
\end{tabular}

Panel B: Output for the improved MSW model with the price change threshold, 2.75. Positive and negative jump sizes obey Lognormal and Exponential laws, respectively.

\begin{tabular}{lrrrrrr}
\hline \multirow{2}{*}{ Number of Jumps } & Total & $(+)$ & $(-)$ & Reversion Rates & $\hat{\alpha}_{X}$ & 0.2176 \\
& 149 & 60 & 89 & $\hat{\alpha}_{Y}$ & 0.4448 \\
\hline \multirow{2}{*}{ Jump Distribution Parameters } & $\hat{\mu}^{+}$ & $\hat{\sigma}^{+}$ & $\hat{\lambda}^{+}$ & $\hat{\mu}^{-}$ & $\hat{\lambda}^{-}$ \\
& -1.3515 & 0.3726 & 0.0274 & 0.3316 & 0.0406 \\
\hline \multirow{2}{*}{ Moments of Diffusion Process } & Mean & Std. Dev. & Skewness & Kurtosis & \\
& 0.0002 & 0.0667 & -0.0091 & 3.1792 & \\
\hline
\end{tabular}


distributions for positive and negative jumps with threshold parameter 2.75 gives the best result for Turkish market. This result is justified by not only the model's capacity in matching the key moments in the analysis, but also its overall goodness of fit, which will be reviewed next.

The output for the improved MSW model, presented in Table 5 confirms the expected decline in the number of detected jumps, in turn higher reversion rates in subprocesses. This finding is also compatible with the reference models which report lower jump intensities than the one we have found with the original specification. The estimated Brownian volatility for Turkish market lies in between those estimated by Geman \& Roncoroni (2006) for low and medium pressure markets (COB and PJM). The authors have not made any remark as to normality of the estimated diffusion process. This feature of MSW Model, which is consistent with the assumptions concerning residuals in econometric analysis, is also preserved in the improved version. Yet, the assumption is violated for the $\operatorname{Exp}$ - $\log N$ alternative which suggests probable negative effects caused by higher thresholds.

\subsection{Model Fit and Parameter Stability}

Assessing goodness-of-fit of electricity spot price models in terms of classical metrics, such as Root Mean Squared Error (RMSE), poses serious drawbacks due to jumps. Emphasizing this end, Mayer et al. (2015) have preferred Ansari-Bradley (1960) test, which compares distributions of two series in terms of variances. However, they assert that lack of sufficient data limits number of tests to be conducted since electricity spot price series is considerably short. Facing the same limitation, we choose to examine moment matching and parameter stability properties of the improved model. Among the four compared in Table 6, all moments, but skewness are found to be highly acceptable.

Absolute biases reported in the final column indicate somewhat better performance than Klüppelberg et al. $(2010)^{2}$ which covers a pure application of BKM Model and are also superior to those reported by Geman \& Roncoroni (2006) in terms of overall fit. Like both studies, results confirm skewness as the toughest moment to approximate. Moreover, we include interquartile range (IQR) and interdecile range (IDR) as goodness-of-fit measures. Janczura \& Weron (2010) used these in comparing alternative regime-switching models, asserting these are more robust to outliers than moment-related statistics. They determine that 2-regime models are inadequate in capturing log-prices by observing IQR and IDR differentials falling in the range $0-0.30$. Proposed 3-regime alternatives are shown to improve the fit by providing simulated series in which the two measures differ from their empirical counterparts by $-3 \%-8 \%$ on average.

Along with moment matching, we consider parameter estimation stability as an evaluation criterion

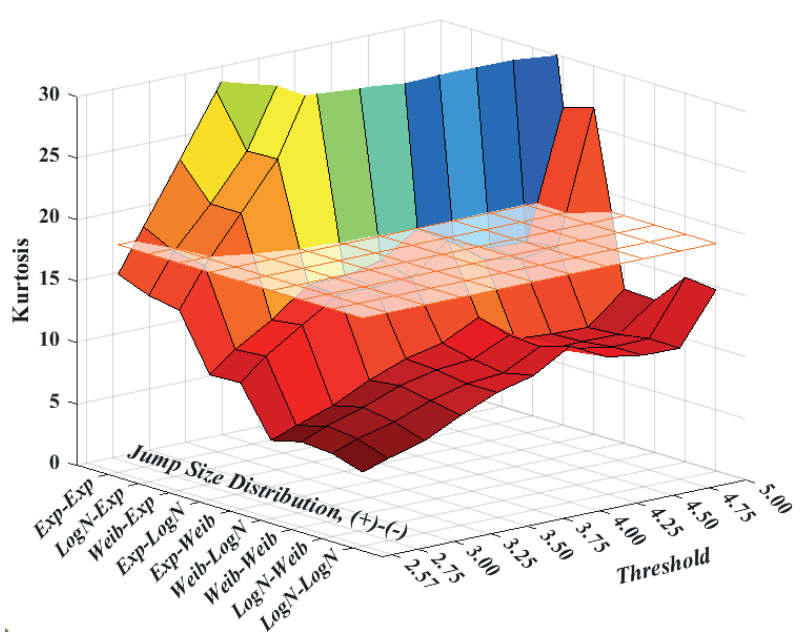

(a) Kurtosis surface plot for simulated series

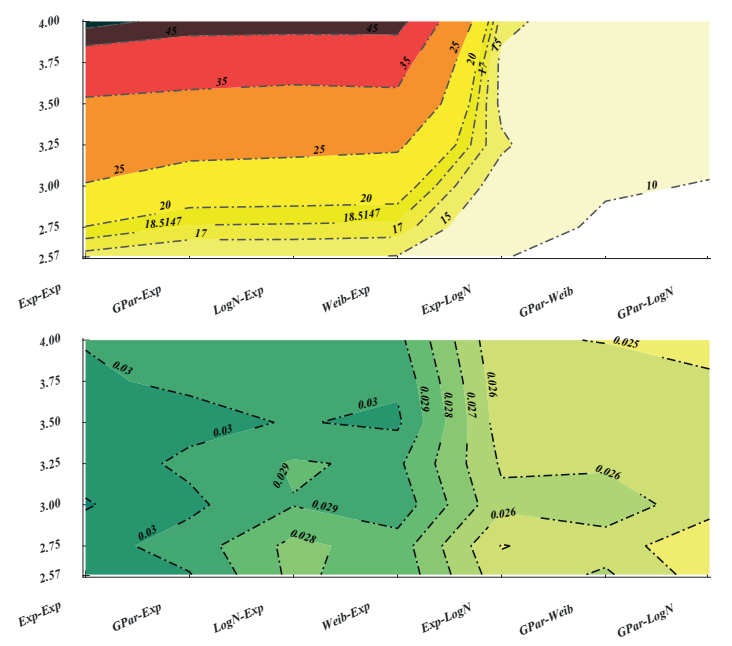

(b) Kurtosis and variance contour plots for simulated series

Figure 4: Mean kurtosis and variance plots of simulated series by selected thresholds and jump size distributions. (Exp: Exponential, LogN: Lognormal, Weib: Weibull, GPar: Generalized Pareto) 
in connection with the quality of simulated processes. While comparable moments and quantiles reflect the model's potential to generate realistic paths, parameter stability refers to robustness of parameters through repeated estimations. We randomly sample 500 series out of the simulated set without replacement for this purpose. Parameter estimations are summarized in Table 6 in terms of their mean values and squared errors with respect to those estimated for $Z_{t}$. These values, with corresponding standard deviations given in parentheses signify good convergence performance of parameters. Mean relative biases, which measure the normalized differences from estimated parameters, are at acceptable levels when compared with the references (e.g., Geman \& Roncoroni, 2006; Klüppelberg et al., 2010). By analysing the biases in jump parameters collectively, we infer that probable misclassification of jump types during simulation might have caused this end. Moreover, further improvements in jump specifications, e.g., introducing truncated version of exponential distribution as in GR Model, are likely to reduce biases, thereby contribute to enhancing more realistic spot price description.

\section{CONCLUSION}

Considering the limited number of studies towards Turkish spot electricity market, we have focused our attention on this developing market. Advanced models in literature realize jumps in price process to be upward directed at initiation. This view reflects the earlier market dynamics that were in charge to a great extent. However, changes in generation mix and policies, and similar factors impose new challenges concerning data generating mechanism. MSW Model has been developed to address such challenges, which are materialized through recent features of price processes. We demonstrate this model can be improved by implementing moment-oriented methodology of advanced models reviewed. Proposed improvement is readily applicable to other markets and is shown to provide better fit. Thus, we contend that this enhancement is likely to serve the intended purpose better, i.e., providing underlying price process for derivatives as communicated by Mayer et al. (2015).

Suggested improvement can also be enhanced with some refinements in simulation and jump specifications. Nevertheless, spot price models are vulnerable to issues which pertain to energy markets, economy, and the environment. Transition to green economy is likely to introduce new complicacies in modelling, which can be overcome in a broader context by scrutinizing interactions with the related markets, like certificates and derivatives markets. As announced by the Turkish market operator EXIST recently, having certificates and futures market online soon will both extend this opportunity and help market develop further.

Structural breaks in seasonality, on the other hand, can have important and wider implications. We note that the break year is a ramification of increased integration of renewable sources and related policies in Turkey, although the motive behind the original suggestion put forth by Nazarova (2014) as to EEX market is different.

Table 6: Statistics of model fit and parameter stability for the improved MSW Model.

Panel A: Comparative moments and IQR and IDR statistics for the distributions of empirical and simulated deseasonalized $\left(Z_{t}\right)$ series.

\begin{tabular}{lllllll}
\hline & Kurtosis & Skewness & Variance & Mean & IQR & IDR \\
\hline Empirical & 18.5147 & -0.9365 & 0.0272 & -0.0120 & 0.1575 & 0.3179 \\
Simulated & 18.5189 & -2.0353 & 0.0278 & -0.0146 & 0.1651 & 0.3288 \\
$|\Delta|(\%)$ & 0.02 & 117.34 & 1.93 & 22.20 & 4.81 & 3.46 \\
\hline
\end{tabular}

Panel B: Statistics for parameter estimation stability. MSE and MRB stand for Mean Squared Error and Mean Relative Bias, respectively.

\begin{tabular}{lllllll}
\hline Parameters & $\begin{array}{l}\text { Model } \\
\text { Values }\end{array}$ & $\widehat{\text { Mean }}$ & $\widehat{\boldsymbol{\sigma}}_{\text {Mean }}$ & $\widehat{\text { MSE }}$ & $\widehat{\boldsymbol{\sigma}}_{\text {MSE }}$ & $\widehat{\text { MRB }}$ \\
\hline$\alpha_{X}$ & 0.2176 & 0.2204 & $(0.0157)$ & 0.0003 & $(0.0004)$ & 0.0126 \\
$\alpha_{Y}$ & 0.4448 & 0.3965 & $(0.0804)$ & 0.0088 & $(0.0079)$ & -0.1085 \\
$\mu^{+}$ & -1.3515 & -1.2540 & $(0.0482)$ & 0.0118 & $(0.0098)$ & -0.0721 \\
$\sigma^{+}$ & 0.3726 & 0.3290 & $(0.0307)$ & 0.0028 & $(0.0028)$ & -0.1170 \\
$\mu^{-}$ & 0.3316 & 0.4745 & $(0.0443)$ & 0.0224 & $(0.0134)$ & 0.4309 \\
\hline
\end{tabular}


Besides increased share of renewables in generation, change in dynamics captured in our case reflect some market participants' rational expectations concerning anticipated increase in FX rates since feed-in tariffs are denominated in USD. This increased share, which are subsidized, imply reduced load to be settled in DAM, in turn lower prices although the overall cost rises. Accordingly, an auction mechanism for renewables is likely to provide cheaper service, which is a leading motive in deregulation. Models, in this regard, has the potential to pinpoint and interpret similar circumstances which deserve attention due to overall impacts on market players, consumers, hence the society.

\section{Disclosure statement}

We declare that we have no conflict of interest to disclose.

\section{Endnotes}

${ }^{1}$ Number of conceivable models jumps to 405 and 7290 with dummy models for weekly seasonality. However, the results suggest Models 2 and 3; and Dummy Models 1 and 2 are superior for macro and weekly seasonality. Like in model space, restricting estimation to certain break years facilitates modeling.

${ }^{2}$ One should take the multiplicative seasonality practice in that paper into account when comparing the first moment. 


\section{REFERENCES}

Aid, R. (2015). Electricity Derivatives. Springer International Publishing AG.

Barndorff-Nielsen, O. E., \& Shephard, N. (2001). Non-Gaussian Ornstein-Uhlenbeck-based models and some of their uses in financial economics. Journal of the Royal Statistical Society: Series B (Statistical Methodology), 63(2), 167-241. https:// doi.org/10.1111/1467-9868.00282

Benth, F. E., Kallsen, J., \& Meyer-Brandis, T. (2007). A non-Gaussian Ornstein-Uhlenbeck process for electricity spot price modeling and derivatives pricing. Applied Mathematical Finance, 14(2), 153-169. https://doi. org/10.1080/13504860600725031

Benth, F. E., Šaltyte-Benth, J., \& Koekebakker, S. (2008). Stochastic Modelling of Electricity and Related Markets. Singapore: World Scientific Publishing Co. Pte. Ltd.

Cartea, Á., \& Figueroa, M. G. (2005). Pricing in Electricity Markets: A Mean Reverting Jump Diffusion Model with Seasonality. Applied Mathematical Finance, 12(4), 313-335.

Clewlow, L., \& Strickland, C. (2000). Energy Derivatives: Pricing and RiskManagement. London: Lacima Publications.

Davis, G. F., \& Kim, S. (2015). Financialization of the Economy. Annual Review of Sociology, 41(1), 203-221. https://doi. org/10.1146/annurev-soc-073014-112402

De Jong, C. (2006). The Nature of Power Spikes: A Regime-Switch Approach. Studies in Nonlinear Dynamics \& Econometrics, 10(3). https://doi.org/10.2202/1558-3708.1361

Edwards, D.W. (2010). Energy Trading and Investing:Trading, Risk Management, and Structruring Deals in the Energy Markets. McGraw-Hill.

Enerji Piyasalari Isletme A.S. (2016). Gun Oncesi Elektrik Piyasasi Piyasa Takas Fiyati Belirleme Yontemi. Retrieved from https://www.epias.com.tr/wp-content/uploads/2016/03/ public_document_v4_released.pdf

European Energy Exchange AG. (2014). EEX Annual Report 2013. Retrieved from https://www.eex.com/ blob/30630/06c68199951eaea397b978d1435f006e/eexgb-2013-en-pdf-data.pdf

European Energy Exchange AG. (2017). EEX Annual Report 2016. Retrieved from https://www.eex.com/blob/66620/17cc36f9c69c14467c7827576dd68db7/eexgb-\%0A2016-enviewer-data.pdf

Geman, H., \& Roncoroni, A. (2006). Understanding the Fine Structure of Electricity Prices. The Journal of Business, 79(3), 1225-1261. https://doi.org/10.1086/500675

Gonzalez, J., Moriarty, J., \& Palczewski, J. (2017). Bayesian calibration and number of jump components in electricity spot price models. Energy Economics, 65, 375-388. https:// doi.org/10.1016/j.eneco.2017.04.022

Grossi, L., Heim, S., Hüschelrath, K., \& Waterson, M. (2018). Electricity market integration and the impact of unilateral policy reforms. Oxford Economic Papers, 70(3), 799-820. https://doi.org/10.1093/oep/gpy005
Harris, C. (2006). Electricity Markets: Pricing, Structures and Economics. West Sussex: John Wiley \& Sons Ltd.

Hayfavi, A., \& Talasli, I. (2014). Stochastic multifactor modeling of spot electricity prices. Journal of Computational and Applied Mathematics, 259, 434-442. https://doi.org/10.1016/j. cam.2013.10.008

Janczura, J., \& Weron, R. (2010). An empirical comparison of alternate regime-switching models for electricity spot prices. Energy Economics, 32(5), 1059-1073. https://doi. org/10.1016/j.eneco.2010.05.008

Klüppelberg, C., Meyer-Brandis, T., \& Schmidt, A. (2010). Electricity spot price modelling with a view towards extreme spike risk. Quantitative Finance, 10(9), 963-974.

Lee, S. S., \& Mykland, P. A. (2008). Jumps in Financial Markets: A New Nonparametric Test and Jump Dynamics. The Review of Financial Studies, 21(6), 2535-2563. https://doi. org/10.1093/rfs/hhm056

Lucia, J. J., \& Schwartz, E. S. (2002). Electricity prices and power derivatives: Evidence from the Nordic Power Exchange. Review of Derivatives Research, 5(1), 5-50. https://doi. org/10.1023/A:1013846631785

Mayer, K., Schmid, T., \& Weber, F. (2015). Modeling electricity spot prices: combining mean reversion, spikes, and stochastic volatility. European Journal of Finance, 21(4), 292-315. https://doi.org/10.1080/1351847X.2012.716775

Meyer-Brandis, T., \& Tankov, P. (2008). Multi-factor jump-diffusion models of electricity prices. International Journal of Theoretical and Applied Finance, 11(5), 503-528. https://doi. org/10.1142/S0219024908004907

Nazarova, A. (2014). Stochastic Models for Energy Markets. Universitat Duisburg-Essen, Essen.

Pirino, D., \& Renò, R. (2010). Electricity Prices: A Nonparametric Approach. International Journal of Theoretical and Applied Finance, 13(2), 285-299.

Popova, J. (2008). Essays on Pricing Electricity and Electricity Derivatives in Deregulated Markets. College of Business and Economics at West Virginia University, Morgantown, West Virginia.

Schwartz, E. S. (1997). The Stochastic Behavior of Commodity Prices: Implications for Valuation and Hedging. The Journal of Finance, 52(3), 923-973.

Seifert, J., \& Uhrig-Homburg, M. (2007). Modelling jumps in electricity prices: theory and empirical evidence. Review of Derivatives Research, 10(1), 59-85.

Shahidehpour, M., Yamin, H., \& Li, Z. (2002). Market Operations in Electric Power Systems: Forecasting, Scheduling, and Risk Management. New York: John Wiley \& Sons, Inc.

Talasli, I. (2012). Stochastic Modeling of Electricity Markets. Middle East Technical University, Ankara, Turkey.

Tokyay, M. B., \& Ozdemir, I. S. (2013). Turkiye Elektrik Piyasasi'nda Elektrik Ticareti. 